Article

\title{
Qualitative Analyses of Integro-Fractional Differential Equations with Caputo Derivatives and Retardations via the Lyapunov-Razumikhin Method
}

\author{
Osman Tunç ${ }^{1}\left(\mathbb{D}\right.$, Özkan Atan $^{2}$ (D), Cemil Tunç ${ }^{3}$ (D) and Jen-Chih Yao ${ }^{4, *}$ \\ 1 Department of Computer Programing, Baskale Vocational School, Van Yuzuncu Yil University, \\ 65080 Van, Turkey; osmantunc89@gmail.com \\ 2 Department of Electric-Electronic Engineering, Faculty of Engineering, Van Yuzuncu Yil University, \\ 65080 Van, Turkey; oatan@yyu.edu.tr \\ 3 Department of Mathematics, Faculty of Sciences, Van Yuzuncu Yil University, 65080 Van, Turkey; \\ cemtunc@yahoo.com \\ 4 Research Center for Interneural Computing, China Medical University Hospital, China Medical University, \\ Taichung 406, Taiwan \\ * Correspondence: yaojc@mail.cmu.edu.tw
}

check for

updates

Citation: Tunç, O.; Atan, Ö.; Tunç, C.; Yao, J.-C. Qualitative Analyses of Integro-Fractional Differential Equations with Caputo Derivatives and Retardations via the Lyapunov-Razumikhin Method. Axioms 2021, 10, 58. https://doi.org/ $10.3390 /$ axioms 10020058

Academic Editors: Wei-Shih Du, Luigi Muglia and Adrian Petrusel

Received: 19 March 2021

Accepted: 6 April 2021

Published: 9 April 2021

Publisher's Note: MDPI stays neutral with regard to jurisdictional claims in published maps and institutional affiliations.

Copyright: (c) 2021 by the authors. Licensee MDPI, Basel, Switzerland. This article is an open access article distributed under the terms and conditions of the Creative Commons Attribution (CC BY) license (https:// creativecommons.org/licenses/by/ $4.0 /)$.

\begin{abstract}
The purpose of this paper is to investigate some qualitative properties of solutions of nonlinear fractional retarded Volterra integro-differential equations (FrRIDEs) with Caputo fractional derivatives. These properties include uniform stability, asymptotic stability, Mittag-Leffer stability and boundedness. The presented results are proved by defining an appropriate Lyapunov function and applying the Lyapunov-Razumikhin method (LRM). Hence, some results that are available in the literature are improved for the FrRIDEs and obtained under weaker conditions via the advantage of the LRM. In order to illustrate the results, two examples are provided.
\end{abstract}

Keywords: nonlinear fractional retarded integro-differential equations; uniform stability; asymptotic stability; Mittag-Leffer stability; boundedness; Lyapunov-Razumikhin method

MSC: 34K20; 34K37; 45J05; 45M10

\section{Introduction}

In recent years, a large number of books [1-3] and papers [4-24] have been devoted to the study of various qualitative properties of solutions of scalars and systems of linear and nonlinear Volterra integro-differential equations (IDEs) both without and with delay, and that of some other kinds of differential equations due to their important applications in population growth models, mathematical models of biological species living together, mathematical models in physics, control engineering and signal processing, mathematical models of heat transfer and radiation, standard closed electric RLC circuits, and so on.

In the relevant literature three methods, which are called the second Lyapunov method, Lyapunov-Krasovskii method and Lyapunov-Razumikhin method, come to the forefront to investigate qualitative properties of solutions of linear and nonlinear integro-differential equations both without and with retardation. Among these methods, the second Lyapunov method and Lyapunov-Krasovskil method are extensively used to study various qualitative behaviors of solutions of integro-differential equations of integer order (see, [4-20]). To the best of our knowledge, the Lyapunov-Razumikhin method is less used during that kind of investigation $[23,25,26]$. However, when it is used for the appropriate problems, it is more effective than the other two methods mentioned, the second Lyapunov method and Lyapunov-Krasovskir method. To the best of our knowledge from the relevant literature, the disadvantages of the Lyapunov second method and Lyapunov-Krasovskii method are that both of these methods require the construction or definition of suitable Lyapunov 
function(s) and Lyapunov-Krasovskir functional(s), which can include double integrals and additional terms. The construction of suitable Lyapunov function(s) and LyapunovKrasovskii functional(s) for nonlinear functional differential equations remains an open problem in the literature at this time. This case is known as a disadvantage. In addition, the time derivatives of double integrals leads to stronger conditions for the negative or negative -semi definite of the time derivative(s) of function(s) or functional(s) used as basic tool(s) in the proof(s).

From this point of view, we would like to present the related work of $\mathrm{Du}$ [27]. Indeed, in 1995, the author investigated the uniformly asymptotic stability of trivial solutions of the system of nonlinear RIDEs of the form:

$$
\dot{x}(t)=-f(t, x(t))+g(t, x(t-\tau))+\int_{t-\tau}^{t} h(t, s, x(s)) d s
$$

or its equivalent system

$$
\dot{x}_{i}(t)=-f_{i}(t, x(t))+g_{i}(t, x(t-\tau))+\int_{t-\tau}^{t} h_{i}(t, s, x(s)) d s,(i=1,2, \ldots, n) .
$$

In this paper, we consider the following initial value problem (IVP) for the system of nonlinear fractional retarded Volterra integro-differential equations (FrRIDEs) with Caputo derivative:

$$
\begin{aligned}
{ }_{t_{0}}^{C} D_{t}^{q} x(t)= & -f(t, x(t))+g(t, x(t), x(t-\tau))+\int_{t-\tau}^{t} h(t, s, x(s)) d s \\
& +\int_{t-\rho}^{t} p(t, s, x(s)) d s+q(t, x(t), x(t-\tau), x(t-\rho)), 0<q<1, \\
x\left(t_{0}+\theta\right)= & \phi(\theta), x\left(t_{0}\right)=\phi(0)=x_{0}, \theta \in[-\tau, 0] \cup[-\rho, 0],
\end{aligned}
$$

where $x=\left(x_{1}, \ldots, x_{n}\right)^{T} \in \mathbb{R}^{n}, t \in \mathbb{R}, s \in[-\tau, \infty) \cup[-\rho, \infty), \tau$ and $\rho$ are positive constants, i.e., they are constant retardations, $f=\left(f_{1}, \ldots, f_{n}\right)^{T} \in C\left(\mathbb{R} \times \mathbb{R}^{n}, \mathbb{R}^{n}\right)$, $f_{i}(t, x(t))=f_{i}\left(t, x_{1}(t), \ldots, x_{n}(t)\right), g=\left(g_{1}, \ldots, g_{n}\right)^{T} \in C\left(\mathbb{R} \times \mathbb{R}^{n} \times C_{H}, \mathbb{R}^{n}\right)$, $C_{H}=\left\{\phi: \phi \in C\right.$ and $\left.\|\phi\|_{t_{0}} \leq H<\infty\right\}, g_{i}(t, x(t), x(t-\tau))=g_{i}\left(t, x_{1}(t), \ldots, x_{n}(t), x_{1}(t-\right.$ $\left.\tau), \ldots, x_{n}(t-\tau)\right), h \in C\left(\mathbb{R} \times[-\tau, \infty) \times C_{H}, \mathbb{R}^{n}\right), p \in C\left(\mathbb{R} \times[-\rho, \infty) \times C_{H}, \mathbb{R}^{n}\right)$ and $q \in$ $C\left(\mathbb{R} \times \mathbb{R}^{n} \times C_{H} \times C_{H}, \mathbb{R}^{n}\right)$. It is supposed that $f(t, 0)=0, g(t, 0,0)=0, p(t, s, 0)=0$, and $h(t, s, 0)=0$. Then, the system of Volterra FrRIDEs (2) with a Caputo derivative includes the zero solution, when $q(.) \equiv 0$.

In this article, motivated by the system of nonlinear RIDEs at Equation (1), i.e., the result of $\mathrm{Du}$ [27] (Theorem 4), and those in the bibliography of this paper, we consider the system of nonlinear FrRIDEs at Equation (2) with a Caputo derivative. As indicated above, we plan to investigate the uniformly stability, asymptotic stability, and Mittag-Leffler stability of the zero solution of Equation (2) with $q \equiv 0$, and the boundedness of all solutions of Equation (2) with $q \neq 0$, by using the Razumikhin method (see [25,26,28-30]). It should be noted that the Caputo derivative is applicable to continuously differentiable quadratic Lyapunov functions to study qualitative properties of solutions of fractional differential equations and fractional delay differential equations, etc. (see, for example, [25,26,31-37]).

It is known that the presence of the fractional derivatives in the system requires that we use appropriately defined fractional derivatives of Lyapunov functions. In the literature, four types of fractional derivatives are commonly applied to calculate the derivatives of Lyapunov functions; these are the Caputo fractional derivative, the Caputo fractional Dini derivative, the Riemann-Liouville fractional derivative, and the Grünwald-Letnikov fractional derivative [32,36]. Not all of these will be employed here. The results pre- 
sented below are new contributions to the literature on delay fractional integro-differential equations with Caputo derivatives.

\section{Preliminaries}

We begin by considering a system of fractional retarded differential equations (FrRDEs) with a Caputo derivative of order $q \in(0,1)$ :

$$
{ }_{t_{0}}^{C} D_{t}^{q} x(t)=F\left(t, x_{t}\right), t \in J, J=\left[t_{0}-\tau, T\right), T \leq+\infty, 0 \leq t_{0} \leq t
$$

where $x \in \mathbb{R}^{n}, F(t, \phi) \in J \times C\left([-r, 0], \mathbb{R}^{n}\right), F(t, 0)=0, x\left(t_{0}+s\right)=\phi(s)$ for $s \in[-r, 0]$, $x\left(t_{0}^{+}\right)=\phi(0), \phi \in C\left([-r, 0], \mathbb{R}^{n}\right), r>0$ is the constant retardation. For $\phi \in C\left([-r, 0], \mathbb{R}^{n}\right)$, we use the usual Euclidean norms $\|$. $\|$ and $\|$. $\|_{t_{0}}$ defined by

$$
\left\|x_{t}\right\|=\sup _{-r \leq s \leq 0}|x(t+s)| \text { and }\|\phi\|_{t_{0}}=\sup _{t_{0}-r \leq s \leq t_{0}}\|\phi(s)\|
$$

respectively.

Since the function $F$ is continuous, for any initial data $\left(t_{0}, \phi\right) \in \mathbb{R}^{+} \times C\left([-r, 0], \mathbb{R}^{n}\right)$, the initial value problem for the system of FrRDEs in Equation (4) has at the least one solution $x(t)=x\left(t, t_{0}, \phi\right) \in C^{1}\left(\left[t_{0}, \infty\right), \mathbb{R}^{n}\right)$. If the function $F$ satisfies a Lipschitz condition in $x$, then the solution is unique.

The following lemmas and other concepts are needed in the remainder of this paper.

Firstly, we give Lemma 1, which is a consequence of (Theorem 2) [31].

Lemma 1. Assume that for any initial data $x\left(t_{0}, \phi_{0}\right) \in \mathbb{R}^{+} \times C\left([-\tau, 0], \mathbb{R}^{n}\right)$, the system of FrRIDEs in Equation (2) has a solution. If there exists a Lyapunov function $V$ and strictly increasing $u, v \in C\left(\mathbb{R}^{+}, \mathbb{R}^{+}\right)$with $u(0)=v(0)=0$ and

$$
u(\|x\|) \leq V(t, x) \leq v(\|x\|) \text { for all } t \geq t_{0}-\tau \text { and all } x \in \mathbb{R}^{n}
$$

and such that for any initial data $\left(t_{0}, \phi_{0}\right) \in \mathbb{R}^{+} \times C\left([-\tau, 0], \mathbb{R}^{n}\right)$ and any point $s>t_{0}$ with

$$
V(s+\xi, x(s+\xi))<V(s, x(s)) \text { for all } \xi \in[-\tau, 0),
$$

the inequality

$$
{ }_{t_{0}}^{C} D_{t}^{q} V(t, x(t)) \leq 0 \text { for all } t \in\left(t_{0}, s\right]
$$

holds, then the zero function of Equation (2) with a zero initial condition is uniformly stable.

Lemma 2 ([31]). The zero solution of the FrRDEs in Equation (4) is asymptotically stable if there exist a continuous function $V(t, x)$, continuous increasing and positive definite functions $u, v, \omega$ and a continuous non-decreasing function $p(s)>s$ for $s>0$ such that the following conditions hold for all $t \in J$ :

$$
\begin{aligned}
& V(t, 0) \equiv 0, u(|x|) \leq V(t, x) \leq v(|x|) \text { for all } t \in J \text { and all } x \in \mathbb{R}^{n} \\
& { }_{t_{0}}^{C} D_{t}^{q} V(t, x(t)) \leq-\omega(|x(t)|) \text { for all } t \in\left(t_{0}, s\right]
\end{aligned}
$$

and

$$
V(t+s, x(t+s))<p V(t, x(t)) \text { for all } s \in[-\tau, 0]
$$

Lemma 3 ([38] Lemma 1). Let $x(t) \in \mathbb{R}^{n}$ be a vector of differentiable functions. Then for any $t \geq t_{0}$,

$$
{ }_{2^{\frac{1}{t_{o}}}} D_{t}^{q}\left(x^{T} x\right) \leq x^{T}(t)_{t_{o}}^{C} D_{t}^{q} x(t) \text { for all } q \in(0,1]
$$


Lemma 4 ([38] Lemma 4). Let $x(t) \in \mathbb{R}^{n}$ be a vector of differentiable functions. Then, for any $t \geq t_{0}$,

$$
{ }_{2^{t_{0}}} D_{t}^{q}\left(x^{T} P x\right) \leq x^{T}(t) P_{t_{0}}^{C} D_{t}^{q} x(t) \text { for all } q \in(0,1],
$$

where $P \in \mathbb{R}^{n \times n}$ is a constant, symmetric and positive definite matrix.

Definition 1 ([35] Definiton 3.1). The trivial solution of the system of FrRIDEs in Equation (2) is said to be Mittag-Leffler stable provided the solution $x(., \phi)$ of (2) satisfies

$$
\|x(t, \phi)\| \leq\left[m\left(\|\phi\|_{\infty}\right) E_{q}\left(-\lambda\left(t-t_{0}\right)^{q}\right)\right]^{b},
$$

where $q \in(0,1), \lambda \geq 0, b>0$,

$$
\|\phi\|_{\infty}=\max _{\theta \in[-\tau, 0]}\|\phi(\theta)\|,
$$

$m(0)=0, m$ is a locally Lipschitz function and is non-negative, and

$$
E_{q}(z)=\sum_{k=0}^{\infty} \frac{z^{k}}{\Gamma(q k+1)}
$$

is the one-parameter Mittag-Leffler function, and $\Gamma$ denotes the Gamma function.

Lemma 5 ([35] Lemma 2.1). Let $x \in \mathbb{R}^{n}$ be a vector of differentiable functions. If a continuous function $V:\left[t_{0}, \infty\right) \times \mathbb{R}^{n} \rightarrow \mathbb{R}^{+}$satisfies

$$
{ }_{t_{0}}^{C} D_{t}^{q} V(t, x(t)) \leq-\alpha V(t, x(t)),
$$

then

$$
V(t, x(t)) \leq V\left(t_{0}, x\left(t_{0}\right)\right) E_{q}\left(-\alpha\left(t-t_{0}\right)^{q}\right),
$$

where $\alpha>0$ and $0<q<1$.

Lemma 6 ([33] Property 1).

$$
{ }_{t_{0}}^{C} D_{t}^{q}(a x(t)+b y(t))=a_{t_{0}}^{C} D_{t}^{q} x(t)+b_{t_{o}}^{C} D_{t}^{q} y(t),
$$

where $q \in(0,1]$.

The contents of the next lemma are well known.

Lemma 7. Let $x \in \mathbb{R}^{n}, n \in N, n \geq 1$, and $M \in \mathbb{R}^{n \times n}$ be a positive definite symmetric $n \times n$ matrix such that

$$
\lambda_{M} \geq \lambda_{i}(M) \geq \lambda_{m},(i=1,2, \ldots, n),
$$

where $\lambda_{i}(M)$ denotes the eigenvalues of $M$. Then

$$
\lambda_{M}\|x\|^{2} \geq\langle M x, x\rangle \geq \lambda_{m}\|x\|^{2},
$$

where $\lambda_{M}$ and $\lambda_{m}$ are the greatest and least eigenvalues of the matrix $M$, respectively.

We know that $\lambda_{M}$ and $\lambda_{m}$ are real and positive since $M$ is a positive definite symmetric matrix.

\section{Razumikhin Analyses of Solutions}

In the system of Volterra FrRIDEs in Equation (2), let $q \equiv 0$, i.e., we consider the system in Equation (2) with Equation (3) replaced by 


$$
\begin{aligned}
{ }_{t_{0}}^{C} D_{t}^{q} x(t)= & -f(t, x(t))+g(t, x(t), x(t-\tau))+\int_{t-\tau}^{t} h(t, s, x(s)) d s \\
& +\int_{t-\rho}^{t} p(t, s, x(s)) d s .
\end{aligned}
$$

We will use the following hypotheses in our main results.

\section{Hypothesis 1.}

$$
\begin{aligned}
& f(t, 0)=g(s, 0,0)=h(t, s, 0) \equiv 0, \\
& x_{i} f_{i}(t, x)>0 \text { as } x_{i} \neq 0, \text { for } t \in \mathbb{R}^{+}, \text {all } x \in \mathbb{R}^{n}
\end{aligned}
$$

Hypothesis 2. The functions $H$ and $P$ satisfy the local Lipschitz condition in $x$, with

$$
H(t, s, x) \equiv \int_{t-\tau}^{t}\|h(t, s, x(s))\| d s, h(t, s, 0)=0,\|h(t, s, x(s))\| \leq h_{0}\|x\| \text { for } s \leq t
$$

and

$$
P(t, s, x) \equiv \int_{t-\rho}^{t}\|p(t, s, x(s))\| d s, p(t, s, 0)=0,\|p(t, s, x(s))\| \leq p_{0}\|x\| \text { for } s \leq t,
$$

where $h_{0}>0, p_{0}>0, h_{0}, p_{0} \in \mathbb{R}$;

\section{Hypothesis 3.}

$$
\begin{gathered}
\|f(t, x)\|-\|g(t, x, x(t-\tau))\|-\left(\tau h_{0}+\rho p_{0}\right)\|x\| \geq 0 \text { for } t \in \mathbb{R}^{+} \\
\text {and all } x \in \mathbb{R}^{n}, x(t-\tau) \in C_{H}
\end{gathered}
$$

\section{Hypothesis 4.}

$$
\begin{gathered}
\|f(t, x)\|-\|g(t, x, x(t-\tau))\|-\left(\tau h_{0}+\rho p_{0}\right)\|x\| \geq \rho_{1}\|x\| \text { for } t \in \mathbb{R}^{+} \\
\text {and all } x \in \mathbb{R}^{n}, x(t-\tau) \in C_{H}, \text { where } \rho_{1}>0, \rho_{1} \in \mathbb{R}
\end{gathered}
$$

Hypothesis 5. There exists $q_{0} \in C\left(\mathbb{R}^{+}, \mathbb{R}\right)$ such that

$$
\begin{gathered}
\|q(t, x, x(t-\tau), x(t-\rho))\| \leq\left|q_{0}(t)\right|\|x\| \text { for all } t \geq t_{0}, x \in \mathbb{R}^{n}, x(t-\tau), \\
x(t-\rho) \in C_{H}
\end{gathered}
$$

and

$$
\begin{gathered}
\|f(t, x)\|-\|g(t, x, x(t-\tau))\|-\left(q_{0}(t)+\tau h_{0}+\rho p_{0}\right)\|x\| \geq 0 \text { for } t \in \mathbb{R}^{+} \\
\text {and all } x \in \mathbb{R}^{n}, x(t-\tau) \in C_{H} .
\end{gathered}
$$

Theorem 1. The zero solution of the system of FrRIDEs in Equation (5) with Caputo derivative is uniformly stable if the conditions of Hypotheses 1-3 hold.

Proof. We define a Lyapunov function $W:=W(t, x)=W(t, x(t))$ by

$$
W(t, x):=\|x\|=\sum_{i=1}^{n}\left|x_{i}\right|=\left|x_{1}\right|+\ldots+\left|x_{n}\right| .
$$


For arbitrary initial data $\left(t_{0}, \phi\right) \in \mathbb{R}^{+} \times C\left([-\tau, 0] \cup[-\rho, 0], \mathbb{R}^{n}\right)$ and a point $t>t_{0}$, it follows that $W(t, x)$ satisfies the Razumikhin condition (see [28-30])

$$
W(t, x(t))>W(t+s, x(t+s))
$$

on the initial set $[-\tau, 0] \cup[-\rho, 0]$, i.e.,

$$
\|x(t)\|>\|x(t+s)\| \text { for all } s \in[-\tau, 0] \cup[-\rho, 0] .
$$

Let $x(t)=x\left(t, t_{0}, \phi\right)$ denote the solution of the IVP of Equation (5) such that $x\left(t_{0}^{+}+s\right)=$ $\phi(s)$ for $s \in[-\tau, 0] \cup[-\rho, 0]$. From this point, it is clear that $W(t, x)$ in Equation (6) satisfies the relations

$$
W(t, 0)=0, \frac{1}{2}\left|x_{1}\right|+\ldots+\frac{1}{2}\left|x_{n}\right|=\frac{1}{2}\|x\| \leq W(t, x),
$$

and

$$
W(t, x) \leq \frac{5}{4}\left|x_{1}\right|+\ldots+\frac{5}{4}\left|x_{n}\right|=\frac{5}{4}\|x\| .
$$

Taking the Caputo fractional derivative of the Lyapunov function $W(t, x)$ in Equation (6) along the system of FrRIDEs in Equation (5), making use the conditions of Hypotheses 1 and 2 and some elementary calculations, we obtain

$$
\begin{aligned}
{ }_{t_{0}}^{C} D_{t}^{q} W(t, x(t))= & { }_{t_{0}}^{C} D_{t}^{q}\left(\left|x_{1}(t)\right|+\left|x_{2}(t)\right|+\ldots+\left|x_{n}(t)\right|\right) \\
= & t_{0}^{C} D_{t}^{q}\left|x_{1}(t)\right|+{ }_{t_{0}}^{C} D_{t}^{q}\left|x_{2}(t)\right|+\ldots+{ }_{t_{0}}^{C} D_{t}^{q}\left|x_{n}(t)\right| \\
= & \left(\operatorname{sign} x_{1}(t)\right)_{t_{0}}^{C} D_{t}^{q} x_{1}(t)+\left(\operatorname{signx} x_{2}(t)\right)_{t_{0}}^{C} D_{t}^{q} x_{2}(t)+\ldots+\left(\operatorname{sign} x_{n}(t)\right)_{t_{0}}^{C} D_{t}^{q} x_{n}(t) \\
= & \sum_{i=1}^{n} x_{i}(t)_{t_{o}}^{C} D_{t}^{q} x_{i}(t) \\
= & \sum_{i=1}^{n} x_{i}(t)\left[-f_{i}(t, x(t))+g_{i}(t, x(t), x(t-\tau))\right] \\
& +\sum_{i=1}^{n} x_{i}(t)\left[\int_{t-\tau}^{t} h_{i}(t, s, x(s)) d s+\int_{t-\rho}^{t} p_{i}(t, s, x(s)) d s\right] \\
\leq & \sum_{i=1}^{n}\left[-\left|f_{i}(t, x(t))\right|+\left|g_{i}(t, x(t), x(t-\tau))\right|\right] \\
& +\sum_{i=1}^{n}\left[\int_{t-\tau}^{t}\left|h_{i}(t, s, x(s))\right| d s+\int_{t-\rho}^{t}\left|p_{i}(t, s, x(s))\right| d s\right] \\
= & -\|f(t, x(t))\|+\|g(t, x(t), x(t-\tau))\|+\int_{t-\tau}^{t}\|h(t, s, x(s))\| d s \\
& +\int_{t-\rho}^{t}\|p(t, s, x(s))\| d s \\
\leq & -\|f(t, x(t))\|+\|g(t, x(t), x(t-\tau))\| \\
& +h_{0} \int_{t-\tau}^{t}\|x(s)\| d s+p_{0}^{t} \int_{t-\rho}^{t}\|x(s)\| d s . \\
&
\end{aligned}
$$


Consider the integral terms such that

$$
h_{0} \int_{t-\tau}^{t}\|x(s)\| d s+p_{0} \int_{t-\rho}^{t}\|x(s)\| d s
$$

which are included in the inequality of Equation (7).

Letting $s-t=\xi$ gives $d s=d \xi$. Hence, for $s=t-\tau$ and $s=t$, it follows that $\xi=-\tau$ and $\xi=0$, respectively. Similarly, by the same transformation and way, for $s=t-\rho$ and $s=t$, we have $\xi=-\rho$ and $\xi=0$, respectively. In view of these estimates, using the Razumikhin condition on the set $s \in[-\tau, 0] \cup[-\rho, 0]$, we get

$$
\begin{aligned}
h_{0} \int_{t-\tau}^{t}\|x(s)\| d s+ & p_{0} \int_{t-\rho}^{t}\|x(s)\| d s=h_{0} \int_{-\tau}^{0}\|x(t+\xi)\| d \xi+p_{0} \int_{-\rho}^{0}\|x(t+\xi)\| d \xi \\
& <h_{0} \int_{-\tau}^{0}\|x(t)\| d \xi+p_{0} \int_{-\rho}^{0}\|x(t)\| d \xi \\
& =h_{0}\|x(t)\| \int_{-\tau}^{0} d \xi+p_{0}\|x(t)\| \int_{-\rho}^{0} d \xi \\
& =h_{0} \tau\|x(t)\|+p_{0} \rho\|x(t)\| .
\end{aligned}
$$

Then, from Equations (7) and (8), it follows that

$$
{ }_{t_{0}}^{C} D_{t}^{q} W(t, x(t)) \leq-\left[\|f(t, x(t))\|-\|g(t, x(t), x(t-\tau))\|-\left(\tau h_{0}+\rho p_{0}\right)\|x(t)\|\right] \leq 0,
$$

that is, using the condition (H3), we have

$$
{ }_{t_{0}}^{C} D_{t}^{q} W(t, x(t)) \leq 0
$$

Thus, from Lemma 1, the zero solution of the system of FrRIDEs in Equation (5) is uniformly stable.

Our next result deals with the asymptotic stability of the system in Equation (5).

Theorem 2. The zero solution of the system of FrRIDEs in Equation (5) is asymptotically stable if the conditions of Hypotheses 1, 2 and 4 hold.

Proof. With $W(t, x)$ defined as in Equation (6), from the conditions (H1), (H2), and (H4) we easily conclude that

$$
\frac{1}{2}\|x\| \leq W(t, x) \leq \frac{5}{4}\|x\|
$$

and

$$
{ }_{t_{0}}^{C} D_{t}^{q} W(t, x(t)) \leq-\rho_{1}\|x(t)\| .
$$

Hence, the zero solution of the system of FrRIDEs in Equation (5) is asymptotically stable by Lemma 2 .

The following theorem shows the Mittag-Leffler stability of the system FrRIDEs in Equation (5).

Theorem 3. The zero solution of the system of FrRIDEs in Equation (5) is Mittag-Leffler stable if the conditions of Hypotheses 1, 2 and 4 hold 
Proof. Again with the Lyapunov function $W(t, x)$ defined as in Equation (6), from the conditions of Hypotheses 1, 2 and 4, it is clear that

$$
{ }_{t_{0}}^{C} D_{t}^{q} W(t, x(t)) \leq-\rho_{1}\|x(t)\|=-\rho_{1} W(t, x(t))
$$

holds.

Using Lemma 5, we obtain

$$
\begin{aligned}
\|x(t)\| & =W(t, x(t)) \leq W\left(t_{0}, x\left(t_{0}\right)\right) E_{q}\left(-\rho_{1}\left(t-t_{0}\right)^{q}\right) \\
& =\left\|x\left(t_{0}\right)\right\| E_{q}\left(-\rho_{1}\left(t-t_{0}\right)^{q}\right) \\
& =\left[m\left(x\left(t_{0}\right)\right) E_{q}\left(-\rho_{1}\left(t-t_{0}\right)^{q}\right)\right] \\
& \leq\left[m\left(\|\phi\|_{\infty}\right) E_{q}\left(-\rho_{1}\left(t-t_{0}\right)^{q}\right)\right]
\end{aligned}
$$

with $m(x)=\|x(t)\|$, which is locally Lipschitz. Thus, the proof of Theorem 3 is completed by using Definition 1 .

\section{Boundedness of Solutions of System in Equation (2)}

We now turn our attention to the perturbed system in Equation (2).

Theorem 4. The solutions of the system of FrRIDEs in Equation (2) are bounded if the conditions of Hypotheses 1, 2 and 5 hold.

Proof. We again consider the Lyapunov function defined in Equation (6). Calculating the time derivative of the Lyapunov function $W(t, x)$ along the system of FrRIDEs in Equation (2) and using the conditions in Hypotheses 1, 2 and 5, we obtain

$$
\begin{aligned}
{ }_{t_{0}}^{C} D_{t}^{q} W(t, x(t)) \leq & -\left[\|f(t, x(t))\|-\|g(t, x(t), x(t-\tau))\|-\left(\tau h_{0}+\rho p_{0}\right)\right]\|x\| \\
& +\|q(t, x, x(t-\tau), x(t-\rho))\| \\
\leq & -\|f(t, x(t))\|+\|g(t, x(t), x(t-\tau))\|+\left(q(t)+\tau h_{0}+\rho p_{0}\right)\|x\| \leq 0 .
\end{aligned}
$$

Hence, we have

$$
W(t, x(t)) \leq W\left(t_{0}, \phi\left(t_{0}\right)\right) .
$$

As a result of this inequality, it follows that

$$
\begin{aligned}
W(t, x(t)) & =\|x(t)\|=\left|x_{1}(t)\right|+\ldots+\left|x_{n}(t)\right| \\
& \leq\left\|x\left(t_{0}\right)\right\|=\left|x_{1}\left(t_{0}\right)\right|+\ldots+\left|x_{n}\left(t_{0}\right)\right|=W\left(t_{0}, \phi\left(t_{0}\right)\right) .
\end{aligned}
$$

Let

$$
K_{0}=\left\|x\left(t_{0}\right)\right\|=\left|x_{1}\left(t_{0}\right)\right|+\ldots+\left|x_{n}\left(t_{0}\right)\right| .
$$

Hence, we obtain

$$
\|x(t)\|=\left|x_{1}(t)\right|+\ldots+\left|x_{n}(t)\right| \leq K_{0} \text { for } t \in \mathbb{R}^{+} .
$$

Hence, it is clear that if $t \rightarrow \infty$, then $\|x(t)\| \leq K_{0}$. This inequality completes the proof of Theorem 4.

Remark 1. Here, if $q=1$, the boundedness of solutions as $t \rightarrow \infty$ was proved without using the Gronwall inequality, see Theorem 4. By this fact, we have removed some unnecessary conditions, and we can obtain some boundedness results in the literature under less restrictive conditions (see, for example, [12,13] and the bibliography therein). Here, we will not state the details of the discussions.

We now give the following example and solve the given system using MATLAB software. In fact, the problem was solved using the 4th order Runge-Kutta method in 
MATLAB. Here, the graphs of Figures 1-4 show the behaviors of paths of the solutions of Example 1 for different values of fractional order $q$.

Example 1. Consider the system of nonlinear Volterra IFrRDEs with Caputo derivative of order $q \in(0,1)$ :

$$
\begin{aligned}
\left(\begin{array}{r}
{ }_{t_{0}}^{C} D_{t}^{q} x_{1}(t) \\
{ }_{t_{0}}^{C} D_{t}^{q} x_{2}(t)
\end{array}\right)= & {\left[\begin{array}{r}
12 x_{1}(t)+\frac{x_{1}(t)}{1+t^{2}+x_{1}^{2}(t)} \\
12 x_{2}(t)+\frac{x_{2}(t)}{1+t^{2}+x_{2}^{2}(t)}
\end{array}\right]+\left[\begin{array}{r}
\frac{x_{1}(t)}{1+t^{2}+x_{1}^{2}\left(t-\frac{1}{10}\right)} \\
\frac{x_{2}(t)}{1+t^{2}+x_{2}^{2}\left(t-\frac{1}{10}\right)}
\end{array}\right] } \\
& +\int_{t-\frac{1}{10}}^{t}\left[\begin{array}{r}
\frac{\sin x_{1}(s)}{1+t^{2}+s^{2}+x_{1}^{2}(s)} \\
\frac{\sin x_{2}(s)}{1+t^{2}+s^{2}+x_{2}^{2}(s)}
\end{array}\right] d s+\int_{t-\frac{1}{5}}^{t}\left[\begin{array}{r}
\frac{x_{1}(s)}{1+t^{2}+s^{2}+x_{1}^{2}(s)} \\
\frac{x_{2}(s)}{1+t^{2}+s^{2}+x_{2}^{2}(s)}
\end{array}\right] d s,
\end{aligned}
$$

where $t \geq \frac{1}{10}, \tau=\frac{1}{10}$ and $\rho=\frac{1}{5}$ are the constant retardations and $x(t)=x \in \mathbb{R}^{2}$.

Comparing the system of IFrRDEs with Caputo derivative in Equation (11) with that given by Equation (5), we have the following formulas:

$$
\begin{aligned}
& f(t, x)=f\left(t, x_{1}, x_{2}\right)=\left[\begin{array}{l}
12 x_{1}+\frac{x_{1}}{1+t^{2}+x_{1}^{2}} \\
12 x_{2}+\frac{x_{2}}{1+t^{2}+x_{2}^{2}}
\end{array}\right], \\
& f(t, 0)=f(t, 0,0)=0, \\
& f_{1}\left(t, x_{1}, x_{2}\right)=12 x_{1}+\frac{x_{1}}{1+t^{2}+x_{1}^{2}}, \\
& x_{1} f_{1}\left(t, x_{1}, x_{2}\right)=12 x_{1}^{2}+\frac{x_{1}^{2}}{1+t^{2}+x_{1}^{2}}>0 \text {, } \\
& x_{1} \neq 0 \text {, } \\
& f_{2}\left(t, x_{1}, x_{2}\right)=12 x_{2}+\frac{x_{2}}{1+t^{2}+x_{2}^{2}}, \\
& x_{2} f_{2}\left(t, x_{1}, x_{2}\right)=12 x_{2}^{2}+\frac{x_{2}^{2}}{1+t^{2}+x_{2}^{2}}>0, \\
& x_{2} \neq 0 \text {, } \\
& -x_{1}(t+0) f_{1}\left(t, x_{1}, x_{2}\right)-x_{2}(t+0) f_{2}\left(t, x_{1}, x_{2}\right) \\
& =-12 x_{1} \operatorname{sgn} x_{1}(t+0)-\frac{x_{1} \operatorname{sgn} x_{1}(t+0)}{1+t^{2}+x_{1}^{2}} \\
& -12 x_{2} \operatorname{sgn} x_{2}(t+0)-\frac{x_{2} \operatorname{sgn} x_{2}(t+0)}{1+t^{2}+x_{2}^{2}} \\
& \leq-11\left|x_{1}\right|-11\left|x_{2}\right|=-11\|x\| \leq-\|f(t, x)\|, \\
& g(t, x, x(t-\tau))=g\left(t, x_{1}, x_{2}, x_{1}\left(t-\frac{1}{10}\right), x_{2}\left(t-\frac{1}{10}\right)\right)=\left[\frac{\frac{x_{1}}{1+t^{2}+x_{1}^{2}\left(t-\frac{1}{10}\right)}}{\frac{x_{2}}{1+t^{2}+x_{2}^{2}\left(t-\frac{1}{10}\right)}}\right], \\
& \|g(t, x, x(t-\tau))\|=\left\|g\left(t, x_{1}, x_{2}, x_{1}\left(t-\frac{1}{10}\right), x_{2}\left(t-\frac{1}{10}\right)\right)\right\|=\left\|\left[\frac{\frac{x_{1}}{1+t^{2}+x_{2}^{2}\left(t-\frac{1}{10}\right)}}{\frac{x_{2}}{1+t^{2}+x_{2}^{2}\left(t-\frac{1}{10}\right)}}\right]\right\| \\
& \leq \frac{\left|x_{1}\right|}{1+t^{2}+x_{1}^{2}\left(t-\frac{1}{10}\right)}+\frac{\left|x_{2}\right|}{1+t^{2}+x_{2}^{2}\left(t-\frac{1}{10}\right)}
\end{aligned}
$$




$$
\begin{gathered}
\leq\left|x_{1}\right|+\left|x_{2}\right|=\|x\|, \\
h(t, s, x)=h\left(t, s, x_{1}, x_{2}\right)=\left[\begin{array}{c}
\frac{\sin x_{1}}{1+t^{2}+s^{2}+x_{1}^{2}} \\
\frac{\sin x_{2}}{1+t^{2}+s^{2}+x_{2}^{2}}
\end{array}\right], \\
h(t, s, 0)=h(t, s, 0,0)=0, \\
\|h(t, s, x)\|=\left\|h\left(t, s, x_{1}, x_{2}\right)\right\|=\left\|\left[\begin{array}{l}
\frac{\sin x_{1}}{1+t^{2}+s^{2}+x_{1}^{2}} \\
\frac{\sin x_{2}}{1+t^{2}+s^{2}+x_{2}^{2}}
\end{array}\right]\right\| \\
=\frac{\left|\sin x_{1}\right|}{1+t^{2}+s^{2}+x_{1}^{2}}+\frac{\left|\sin x_{2}\right|}{1+t^{2}+s^{2}+x_{2}^{2}} \\
\leq\left|\sin x_{1}\right|+\left|\sin x_{2}\right| \leq\left|x_{1}\right|+\left|x_{2}\right|=\|x\|,
\end{gathered}
$$

where $h_{0}=1$,

$$
\begin{gathered}
H(t, s, x) \equiv \int_{t-\tau}^{t}\|h(t, s, x(s))\| d s=\int_{t-\frac{1}{10}}^{t}\left\|\left[\frac{\sin x_{1}(s)}{1+t^{2}+s^{2}+x_{1}^{2}(s)}\right]\right\| d s \\
\left.\quad=\int_{t-\frac{1}{10} x_{2}(s)}^{t+t^{2}+s^{2}+x_{2}^{2}(s)}\right] \| \frac{\left|\sin x_{1}(s)\right|}{1+t^{2}+s^{2}+x_{1}^{2}(s)} d s \\
\quad+\int_{t-\frac{1}{10}}^{t} \frac{\left|\sin x_{2}(s)\right|}{1+t^{2}+s^{2}+x_{2}^{2}(s)} d s \\
\leq \int_{t-\frac{1}{10}}^{t}\left|\sin x_{1}(s)\right| d s+\int_{t-\frac{1}{10}}^{t}\left|\sin x_{2}(s)\right| d s \\
\leq \int_{t-\frac{1}{10}}^{t}\left|x_{1}(s)\right| d s+\int_{t-\frac{1}{10}}^{t}\left|x_{2}(s)\right| d s=\int_{t-\frac{1}{10}}^{t}\|x(s)\| d s .
\end{gathered}
$$

Let $s-t=\xi$, which implies $d s=d \xi$. Then, for $s=t-\frac{1}{10}$, we derive $\xi=-\frac{1}{10}$, and similarly for $s=t$, we have $\xi=0$.

In view of these findings, using the given Razumikhin condition [28-30] on the initial segment $\left[-\frac{1}{10}, 0\right]$, it follows that

$$
\int_{t-\frac{1}{10}}^{t}\|x(s)\| d s=\int_{-\frac{1}{10}}^{0}\|x(t+\xi)\| d \xi<\int_{-\frac{1}{10}}^{0}\|x(t)\| d \xi=\frac{1}{10}\|x\| .
$$

For this step, we consider the term:

$$
P(t, s, x) \equiv \int_{t-\rho}^{t}\|p(t, s, x(s))\| d s
$$


with

$$
p(t, s, x)=p\left(t, s, x_{1}, x_{2}\right)=\left[\frac{\frac{x_{1}}{1+t^{2}+s^{2}+x_{1}^{2}}}{\frac{x_{2}}{1+t^{2}+s^{2}+x_{2}^{2}}}\right], \rho=\frac{1}{5} .
$$

In that case, we derive

$$
p(t, s, 0,0)=0
$$

and

$$
\begin{gathered}
\left.\|p(t, s, x)\|=\left\|p\left(t, s, x_{1}, x_{2}\right)\right\|=\|\left[\frac{\frac{x_{1}}{1+t^{2}+s^{2}+x_{1}^{2}}}{\frac{x_{2}}{1+t^{2}+s^{2}+x_{2}^{2}}}\right]\right] \| \\
\quad=\frac{\left|x_{1}\right|}{1+t^{2}+s^{2}+x_{1}^{2}}+\frac{\left|x_{2}\right|}{1+t^{2}+s^{2}+x_{2}^{2}} \\
\leq\left|x_{1}\right|+\left|x_{2}\right|=\|x\|, \text { where } p_{0}=1 .
\end{gathered}
$$

For the next step, it follows that

$$
\begin{aligned}
P(t, s, x) & \equiv \int_{t-\rho}^{t}\|p(t, s, x(s))\| d s=\int_{t-\frac{1}{5}}^{t}\left\|\left[\frac{\frac{x_{1}(s)}{1+t^{2}+s^{2}+x_{1}^{2}(s)}}{\frac{x_{2}(s)}{1+t^{2}+s^{2}+x_{2}^{2}(s)}}\right]\right\| d s \\
& \leq \int_{t-\frac{1}{5}}^{t}\left|x_{1}(s)\right| d s+\int_{t-\frac{1}{5}}^{t}\left|x_{2}(s)\right| d s=\int_{t-\frac{1}{5}}^{t}\|x(s)\| d s .
\end{aligned}
$$

Let $s-t=\xi$, which implies $d s=d \xi$. Then, for $s=t-\frac{1}{5}$, we derive $\xi=-\frac{1}{5}$. Similarly, for $s=t$, we have $\xi=0$. Then,

$$
\int_{t-\frac{1}{5}}^{t}\|x(s)\| d s=\int_{-\frac{1}{5}}^{0}\|x(t+\xi)\| d \xi<\int_{-\frac{1}{5}}^{0}\|x(t)\| d \xi=\frac{1}{5}\|x\| .
$$

Hence, bringing together the above results, we derive

$$
\begin{aligned}
& \|f(t, x)\|-\|g(t, x, x(t-\tau))\|-\left(\tau h_{0}+\rho p_{0}\right)\|x\| \\
& \geq\|f(t, x)\|-\left\|g\left(t, x, x\left(t-\frac{1}{2}\right)\right)\right\|-\left(\frac{1}{10}+\frac{1}{5}\right)\|x\| \\
& \geq 11\|x\|-\|x\|-\frac{3}{10}\|x\|=(9.7)\|x\|, \text { where } \rho_{1}=\frac{97}{10} .
\end{aligned}
$$

In the light of the above discussion, the conditions of Hypotheses 1-3 of Theorem 1, and the conditions of Hypotheses 1, 2 and 4 of Theorems 2 and 3 hold. For this reason, the zero solution of the system of FrRIDEs in Equation (11) with Caputo derivative is uniformly stable, asymptotically stable and Mittag-Leffler stable.

In Figures 1-4, the system of FrRIDEs (11) was solved and the orbits of the solutions $x_{1}(t), x_{2}(t)$ were drawn for $\tau=\frac{1}{10}, \rho=\frac{1}{5}$ and different initial values when $t \geq \frac{1}{10}$. 


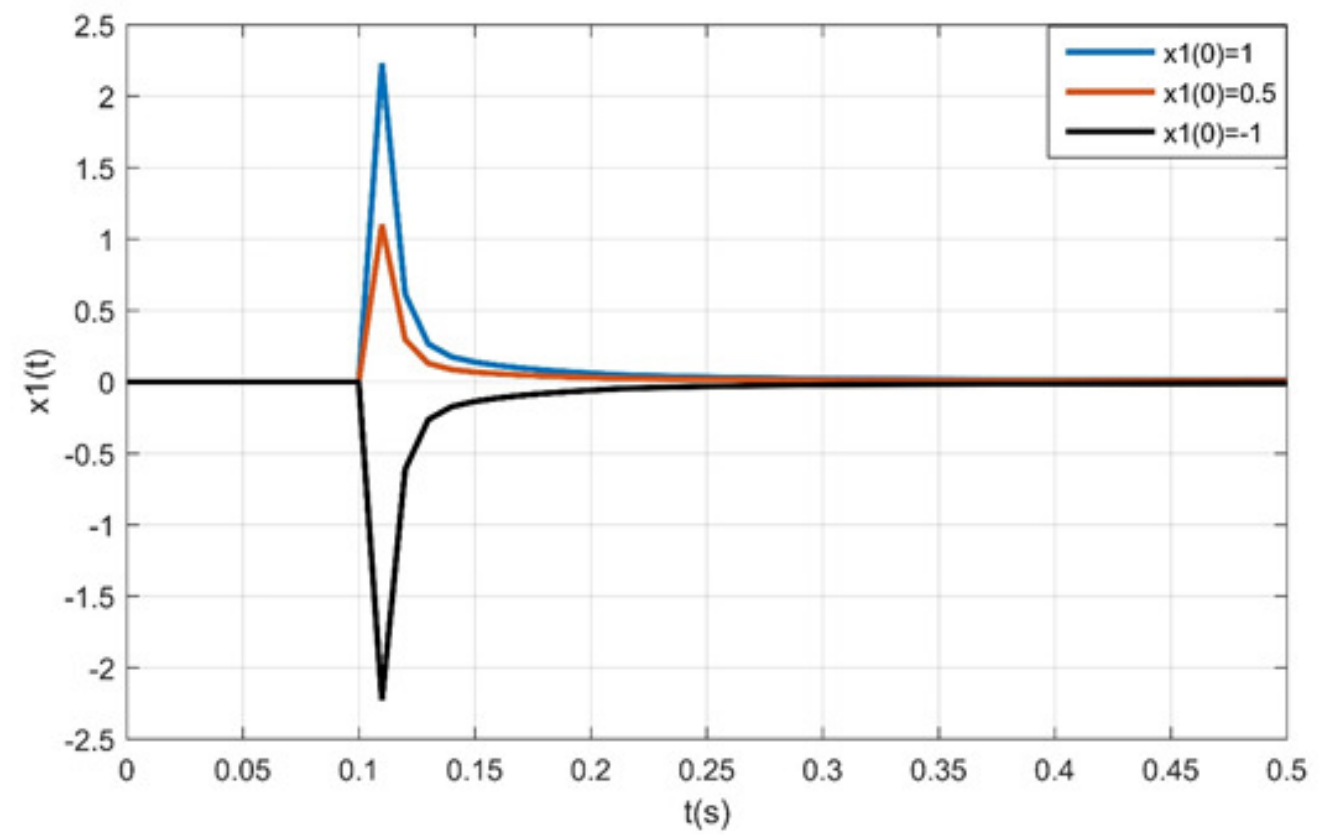

Figure 1. The behaviors of uniformly, asymptotically and Mittag-Leffler stable solution $x_{1}(t)$ of the system of of fractional retarded Volterra integro-differential equations (FrRIDEs) in Equation (11) for $q=0.5, \tau=\frac{1}{10}, \rho=\frac{1}{5}$ and different initial values when $t \geq \frac{1}{10}$.

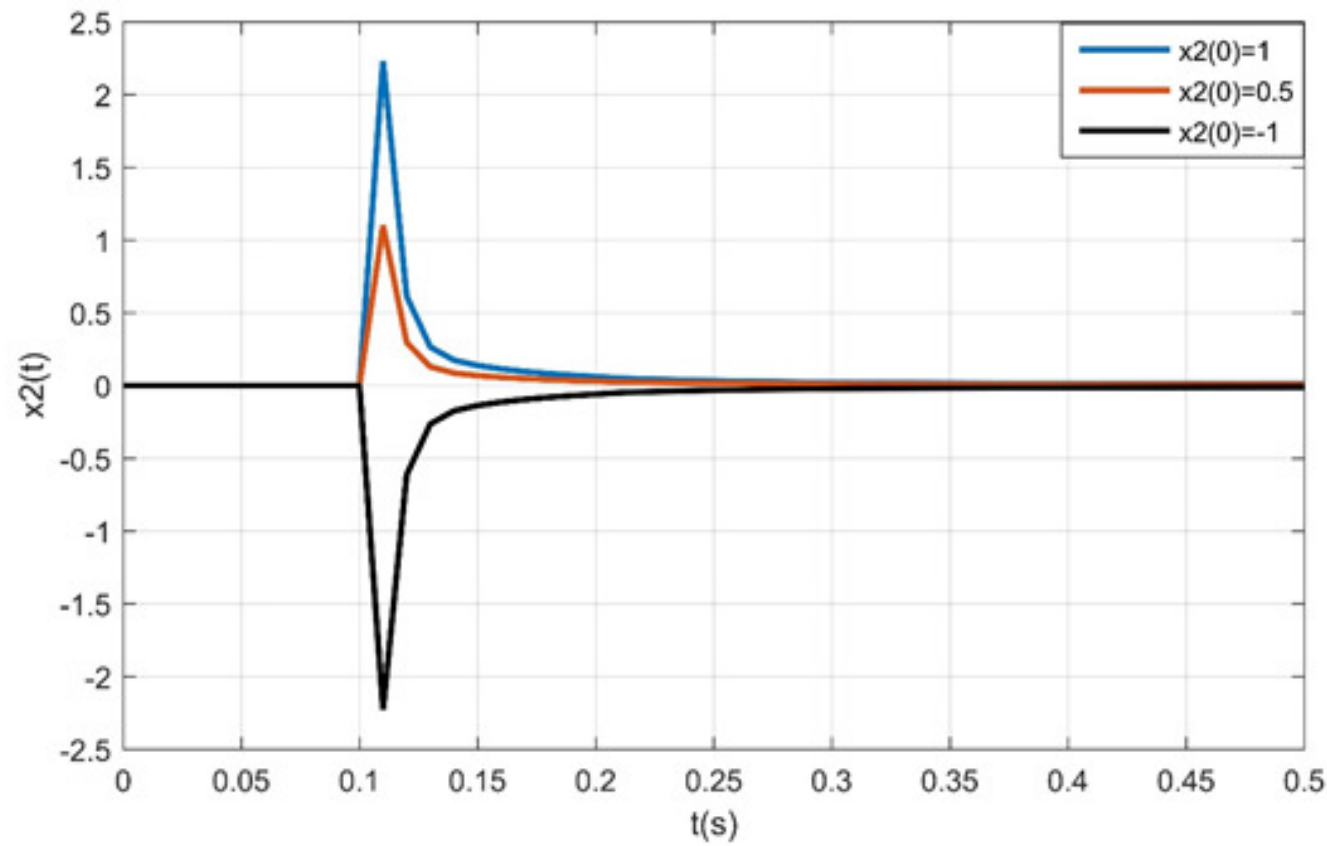

Figure 2. The behaviors of uniformly, asymptotically and Mittag-Leffler stable solution $x_{2}(t)$ of the system of of FrRIDEs in Equation (11) for $q=0.5, \tau=\frac{1}{10}, \rho=\frac{1}{5}$ and different initial values when $t \geq \frac{1}{10}$. 


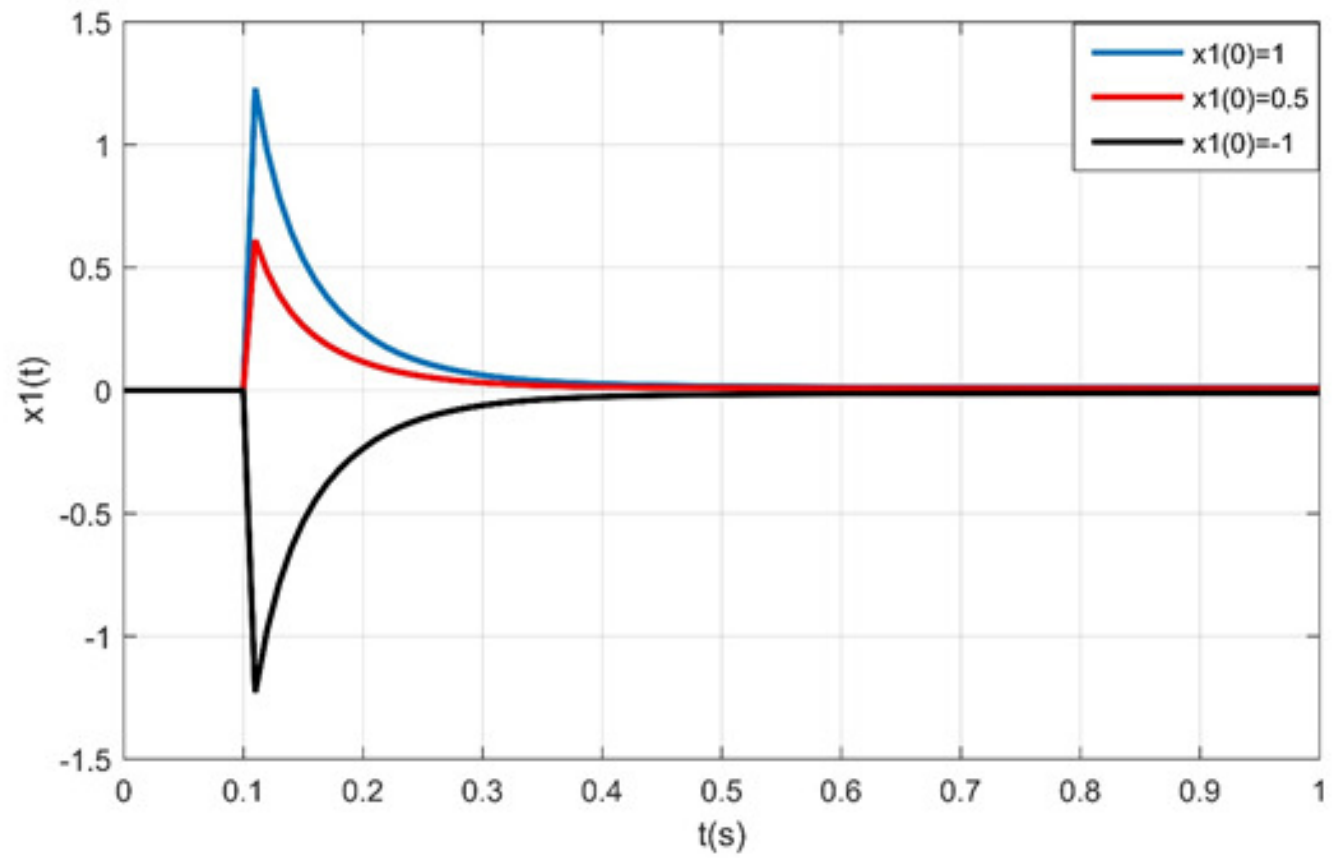

Figure 3. The behaviors of uniformly, asymptotically and Mittag-Leffler stable solution $x_{1}(t)$ of the system of of FrRIDEs in Equation (11) for $q=0.9, \tau=\frac{1}{10}, \rho=\frac{1}{5}$ and different initial values when $t \geq \frac{1}{10}$.

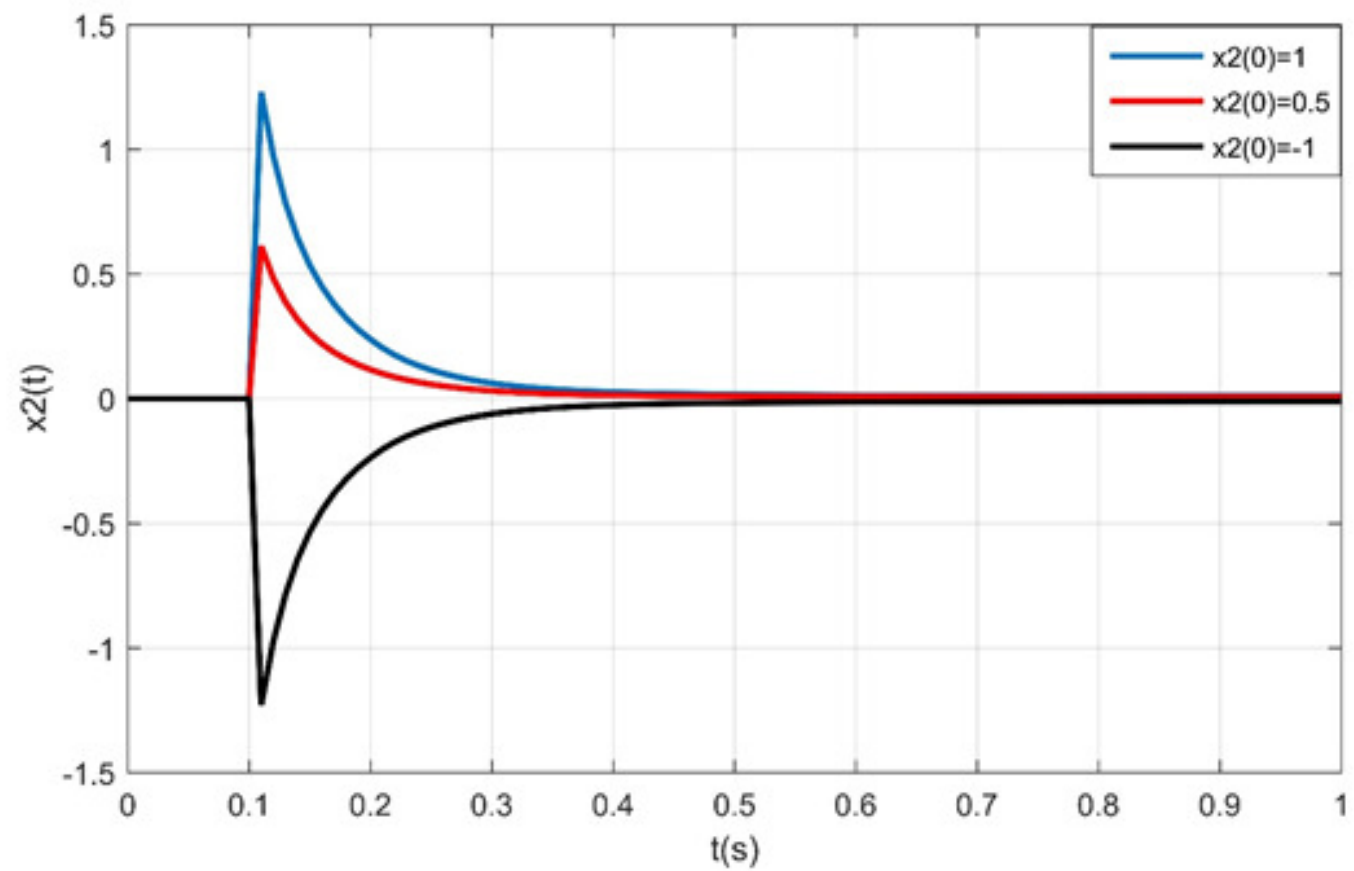

Figure 4. The behaviors of uniformly, asymptotically and Mittag-Leffler stable solution $x_{2}(t)$ of the system of of FrRIDEs in Equation (11) for $q=0.9, \tau=\frac{1}{10}, \rho=\frac{1}{5}$ and different initial values and different initial values when $t \geq \frac{1}{10}$.

For the case $q() \neq$.0 , we now give the second example and solve it using MATLAB software. 
Example 2. Consider the system of nonlinear Volterra FrRIDEs with Caputo derivative of or$\operatorname{der} q \in(0,1)$ :

$$
\begin{aligned}
\left(\begin{array}{c}
C_{t_{0}}^{C} D_{t}^{q} x_{1}(t) \\
C_{t_{0}} D_{t}^{q} x_{2}(t)
\end{array}\right)= & {\left[\begin{array}{c}
12 x_{1}(t)+\frac{x_{1}(t)}{1+t^{2}+x_{1}^{2}(t)} \\
12 x_{2}(t)+\frac{x_{2}(t)}{1+t^{2}+x_{2}^{2}(t)}
\end{array}\right]+\left[\begin{array}{r}
\frac{x_{1}(t)}{1+t^{2}+x_{1}^{2}\left(t-\frac{1}{10}\right)} \\
\frac{x_{2}(t)}{1+t^{2}+x_{2}^{2}\left(t-\frac{1}{10}\right)}
\end{array}\right] } \\
& +\int_{t-\frac{1}{10}}^{t}\left[\begin{array}{r}
\frac{\sin x_{1}(s)}{1+t^{2}+s^{2}+x_{1}^{2}(s)} \\
\frac{\sin x_{2}(s)}{1+t^{2}+s^{2}+x_{2}^{2}(s)}
\end{array}\right] d s+\int_{t-\frac{1}{5}}^{t}\left[\begin{array}{r}
\frac{x_{1}(s)}{1+t^{2}+s^{2}+x_{1}^{2}(s)} \\
\frac{x_{2}(s)}{1+t^{2}+s^{2}+x_{2}^{2}(s)}
\end{array}\right] d s \\
& +\left(\frac{\frac{\exp (t) x_{1}}{1+\exp (2 t)+\left|x_{1}\left(t-\frac{1}{10}\right)\right|+\left|x_{1}\left(t-\frac{1}{5}\right)\right|}}{\left.1+\exp (2 t)+\mid x_{2}(t)-\frac{1}{10}\right)|+| x_{2}\left(t-\frac{1}{5}\right) \mid}\right.
\end{aligned}
$$

where $t \geq \frac{1}{10}, \tau=\frac{1}{10}$ and $\rho=\frac{1}{5}$ are the constant delay terms and $x(t)=x \in \mathbb{R}^{2}$.

Comparing the systems of FrRIDEs in Equation (13) with Caputo derivative and Equation (2), we note that the functions $-f(t, x), g\left(t, x, x\left(t-\frac{1}{10}\right)\right), h(t, s, x)$ and $p(t, s, x)$ are the same as those in Example 1. Then, the satisfaction of the conditions of Hypotheses 1 and 2 have been shown in Example 1. For the verification of the condition of Hypothesis 5, we consider the last term of Equation (13):

$$
q\left(t, x, x\left(t-\frac{1}{10}\right), x\left(t-\frac{1}{5}\right)\right)=\left(\begin{array}{c}
\frac{\exp (t) x_{1}}{1+\exp (2 t)+\left|x_{1}\left(t-\frac{1}{10}\right)\right|+\left|x_{1}\left(t-\frac{1}{5}\right)\right|} \\
\frac{\exp (t) x_{2}}{1+\exp (2 t)+\left|x_{2}\left(t-\frac{1}{10}\right)\right|+\left|x_{2}\left(t-\frac{1}{5}\right)\right|}
\end{array}\right)
$$

Clearly, it follows that

$$
\begin{aligned}
\left|q\left(t, x, x\left(t-\frac{1}{10}\right), x\left(t-\frac{1}{5}\right)\right)\right|= & \left|\left(\frac{\left.\frac{\exp (t) x_{1}}{1+\exp (2 t)+\left|x_{1}\left(t-\frac{1}{10}\right)\right|+\left|x_{1}\left(t-\frac{1}{5}\right)\right|}\right) \mid}{\frac{\exp (t) x_{2}}{1+\exp (2 t)+\left|x_{2}\left(t-\frac{1}{10}\right)\right|+\left|x_{2}\left(t-\frac{1}{5}\right)\right|}}\right)\right| \\
= & \frac{\exp (t)\left|x_{1}\right|}{1+\exp (2 t)+\left|x_{1}\left(t-\frac{1}{10}\right)\right|+\left|x_{1}\left(t-\frac{1}{5}\right)\right|} \\
& +\frac{\exp (t)\left|x_{2}\right|}{1+\exp (2 t)+\left|x_{2}\left(t-\frac{1}{10}\right)\right|+\left|x_{2}\left(t-\frac{1}{5}\right)\right|} \\
\leq & \frac{\exp (t)\left|x_{1}\right|}{1+\exp (2 t)}+\frac{\exp (t)\left|x_{2}\right|}{1+\exp (2 t)} \\
= & \frac{\exp (t)}{1+\exp (2 t)}\left[\left|x_{1}\right|+\left|x_{2}\right|\right]=\left|q_{0}(t)\right|\|x\|,
\end{aligned}
$$

where

$$
\left|q_{0}(t)\right|=\frac{\exp (t)}{1+\exp (2 t)} \leq \frac{1}{2},\left|x_{1}\right|+\left|x_{2}\right|=\|x\| .
$$

In view of Equations (12) and (14), it is clear that

$$
\begin{aligned}
\|f(t, x)\|-\|g(t, x, x(t-\tau))\|-\left(q_{0}(t)+\tau h_{0}+\rho p_{0}\right)\|x\| & \geq(9.7)\|x\|-\frac{\exp (t)}{1+\exp (2 t)}\|x\| \\
& \geq(9.7)\|x\|-\frac{1}{2}\|x\|=(9.2)\|x\| .
\end{aligned}
$$

As a consequence of this inequality, the condition of Hypothesis 5 holds. Thus, the solutions of the system of FrRIDEs in Equation (13) with Caputo derivative are bounded as $t \rightarrow \infty$. 
The following graphs of Figures 5-8 show the behaviors of paths of the solutions of Example 2 for different values of fractional order $q$.

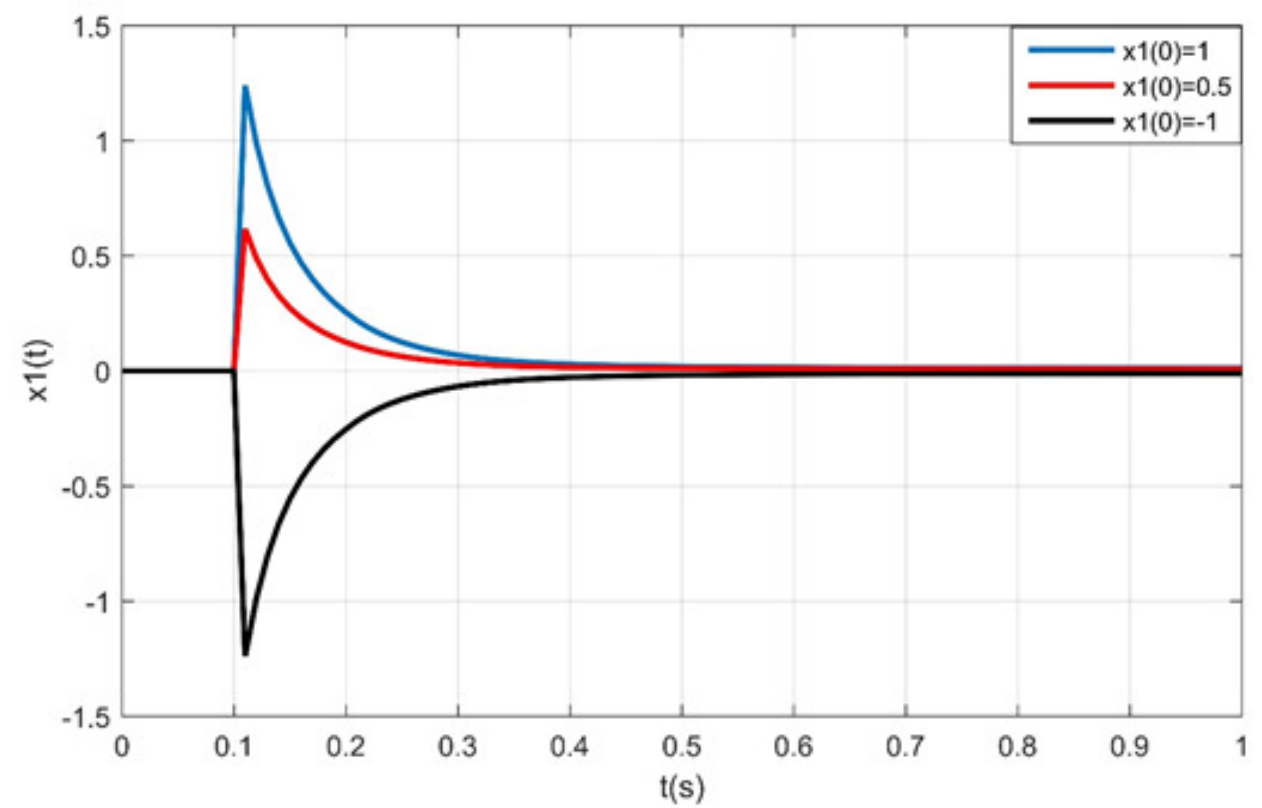

Figure 5. The boundedness of solution $x_{1}(t)$ of the system of IFrRDEs in Equation (13) for $q=0.5$, $\tau=\frac{1}{10}, \rho=\frac{1}{5}$ and different initial values when $t \geq \frac{1}{10}$.

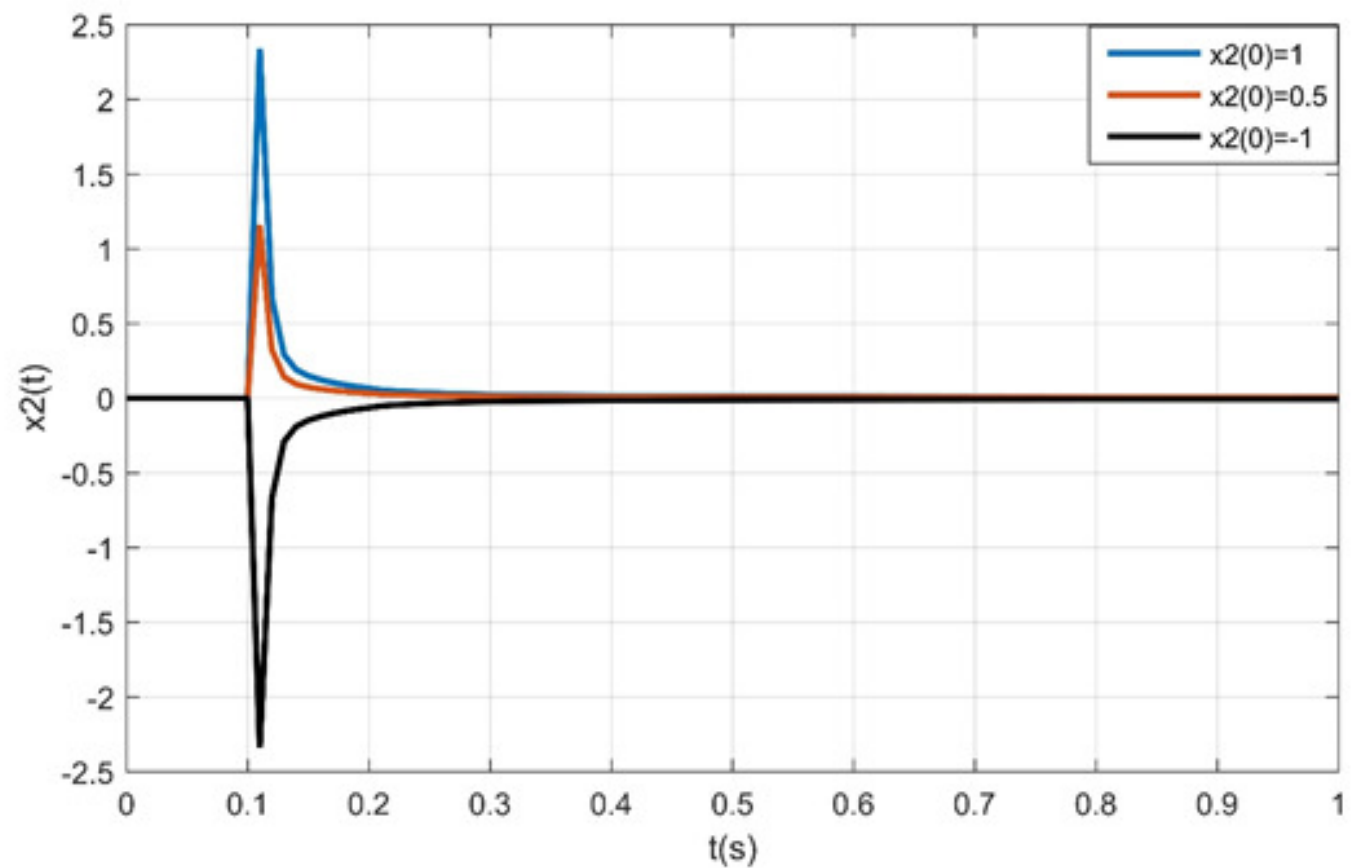

Figure 6. The boundedness of solution $x_{2}(t)$ of the system of IFrRDEs in Equation (13) for $q=0.5$, $\tau=\frac{1}{10}, \rho=\frac{1}{5}$ and different initial values when $t \geq \frac{1}{10}$. 


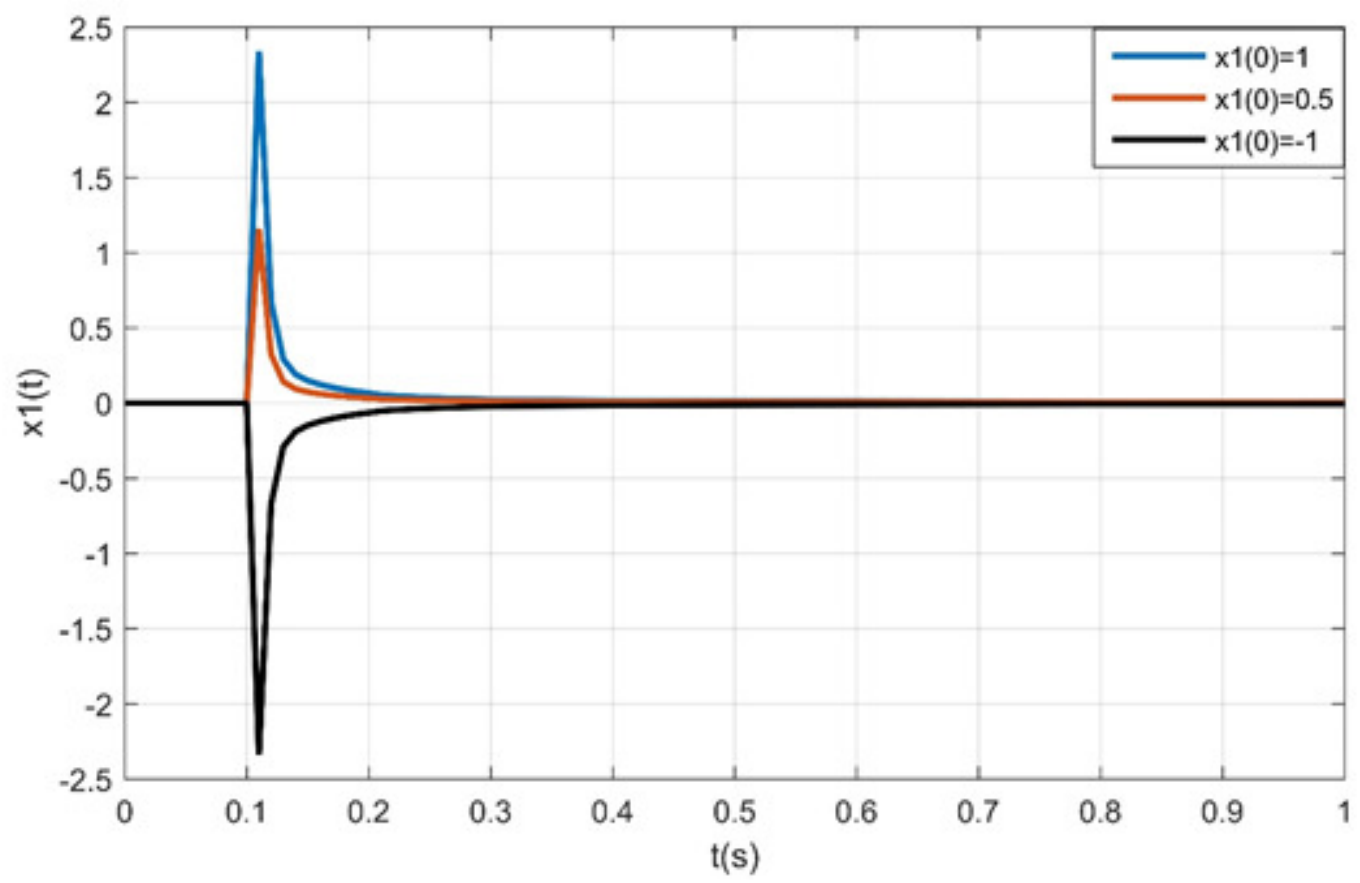

Figure 7. The boundedness of solution $x_{1}(t)$ of the system of IFrRDEs in Equation (13) for $q=0.9$, $\tau=\frac{1}{10}, \rho=\frac{1}{5}$ and different initial values when $t \geq \frac{1}{10}$.

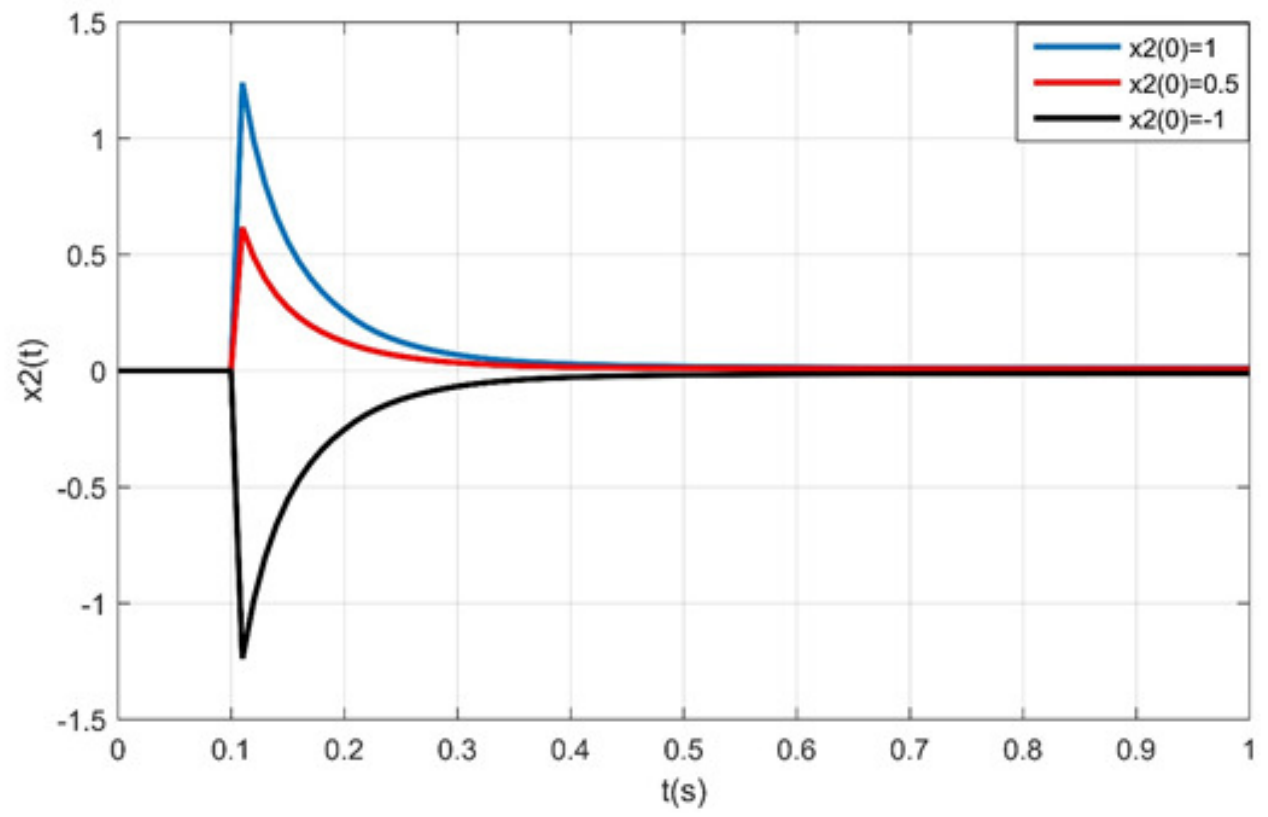

Figure 8. The boundedness of solution $x_{2}(t)$ of the system of IFrRDEs in Equation (13) for $q=0.5$, $\tau=\frac{1}{10}, \rho=\frac{1}{5}$ and different initial values when $t \geq \frac{1}{10}$.

\section{Discussions}

We would like to explain the contributions of this paper to the relevant literature as the following.

(1) To the best of our knowledge, in the literature, there are numerous papers on the uniform stability, asymptotic stability, Mittag-Leffer stability and boundedness of fractional differential equations of integer order both with and without delay. However, there are no papers in the literature on the asymptotic stability, Mittag-Leffer stability and boundedness of the FrRIDEs in Equation (2) with Caputo fractional derivative, except the two papers of Hristova and Tunç $[25,26]$, which include some results on 
the uniform stability. Next, qualitative behaviors of the FrRIDEs in Equation (2) have not been discussed in the literature yet. Therefore, the results of this paper are new, original and they have scientific novelty.

(2) If $q=1$ in the FrRIDEs in Equation (2), then we have the system of RIDEs

$$
\begin{aligned}
\dot{x}(t)= & -f(t, x(t))+g(t, x(t), x(t-\tau))+\int_{t-\tau}^{t} h(t, s, x(s)) d s \\
& +\int_{t-\rho}^{t} p(t, s, x(s)) d s+q(t, x(t), x(t-\tau), x(t-\rho)) .
\end{aligned}
$$

It is clear that the system of RIDEs in Equation (15) includes, extends and improves the system of RIDEs in Equation (1). This is a contribution to the topic and the relevant literature.

(3) In $\mathrm{Du}$ [27] (Theorem 4), the uniform asymptotic stability of the zero solution of the system of RIDEs in Equation (1) was proved using the Lyapunov-Krasovskir functional:

$$
V(t, x(.)):=\|x\|+\int_{t-\tau}^{t}\|g(s, x(s))\| d s+\int_{t-\tau}^{t} \int_{t}^{\infty}\|h(u, s, x(s))\| d u d s .
$$

We can prove the same result, [27] (Theorem 4) using the Lyapunov-Razumikhin method and the Lyapunov function

$$
W(t, x):=\|x\|=\sum_{i=1}^{n}\left|x_{i}\right|=\left|x_{1}\right|+\ldots+\left|x_{n}\right| .
$$

Clearly, this Lyapunov function does not include the term $\int_{t-\tau}^{t}\|g(s, x(s))\| d s$. The time derivative of this term gives

$$
\frac{d}{d t} \int_{t-\tau}^{t}\|g(s, x(s))\| d s=\|g(t, x(t))\|-\|g(t-\tau, x(t-\tau))\| .
$$

Based on this approach, we can obtain the result of $\mathrm{Du}$ [27] (Theorem 4) under weaker conditions. Namely, we remove the following hypothesis from Du [27] (Theorem 4):

$$
\|g(t-\tau, x(t-\tau))\|-\|g(t, x(t-\tau))\| \geq 0, t \in \mathbb{R}^{+} .
$$

To the best of our information, this is a stronger condition and the satisfaction of this hypothesis can be difficult. Removing this condition from that of Du [27] (Theorem 4) leads to an important and strong advantage during the applications of that kind of equation.

(4) Du [27] (Theorem 4) proved the related theorem without giving an example in a particular case, which verifies the hypotheses of [27] (Theorem 4). In this paper, we provided two examples and solved them with MATLAB software, which verifies the applicability of the results of this paper.

\section{Conclusions}

This paper has proposed an effective way to discuss some qualitative properties of solutions of nonlinear Volterra integro-differential equations with Caputo fractional derivatives and multiple constant retardations. Here, a new mathematical model consisting of non-linear fractional Volterra integro-differential equations with Caputo fractional deriva- 
tives and two constant retardations was considered. New sufficient conditions for the uniform stability, asymptotic stability and Mittag-Leffer stability of the zero solution, as well as the boundedness of the solutions were obtained. The presented results were proved by defining an appropriate Lyapunov function and applying the Lyapunov-Razumikhin method. An advantage of the new function and method used here is that they eliminate using Gronwall's inequality. Compared to related results in the literature, the conditions here are new, more general, simple and convenient to apply. Examples to show the application of the theorems have been included.

Author Contributions: Investigation, O.T., Ö.A., C.T. and J.-C.Y. Writing-Original Draft: O.T., Ö.A., C.T. and J.-C.Y. All authors have read andagreed to the published version of the manuscript.

Funding: The research of J. C. Yao was partially supported by the Grant MOST 108-2115-M-039-005MY3.

Institutional Review Board Statement: Not applicable.

Informed Consent Statement: Not applicable.

Data Availability Statement: Not applicable.

Acknowledgments: The authors would like to thank the three anonymous referees and the handling for many useful comments and suggestions, leading to a substantial improvement in the presentation of this article.

Conflicts of Interest: The authors declare no conflict of interest.

\section{References}

1. Burton, T.A. Volterra integral and differential equations. In Mathematics in Science and Engineering, 2nd ed.; Elsevier: Amsterdam, The Netherlands, 2005; p. 202.

2. Rahman, M. Integral Equations and Their Applications; WIT Press: Southampton, UK, 2007.

3. Wazwaz, A.M. Linear and nonlinear integral equations. In Methods and Applications; Higher Education Press: Beijing, China; Springer: Heidelberg, Germany, 2011.

4. Alahmadi, F.; Raffoul, Y.; Alharbi, S. Boundedness and stability of solutions of nonlinear Volterra integro-differential equations. Adv. Dyn. Syst. Appl. 2018, 13, 19-31.

5. Andreev, A.S.; Peregudova, O.A. On the stability and stabilization problems of Volterra integro-differential equations. Russ. J. Nonlinear Dyn. 2018, 14, 387-407. [CrossRef]

6. Berezansky, L.; Domoshnitsky, A. On stability of a second order integro-differential equation. Nonlinear Dyn. Syst. Theory 2019, 19, 117-123.

7. Chang, Y.-K.; Ponce, R. Uniform exponential stability and applications to bounded solutions of integro-differential equations in Banach spaces. J. Integr. Equ. Appl. 2018, 30, 347-369. [CrossRef]

8. Anh, N.P.H.; Tran, A. The New stability criteria for nonlinear Volterra integro-differential equations. Acta Math. Vietnam. 2018, 43, 485-501.

9. Raffoul, Y.; Rai, H. Uniform stability in nonlinear infinite delay Volterra integro-differential equations using Lyapunov functionals. Nonauton. Dyn. Syst. 2016, 3, 14-23. [CrossRef]

10. Tunç, C. Properties of solutions to Volterra integro-differential equations with delay. Appl. Math. Inf. Sci. 2016, 10, 1775-1780. [CrossRef]

11. Tunç, C. Qualitative properties in nonlinear Volterra integro-differential equations with delay. J. Taibah Univ. Sci. 2017, 11, 309-314. [CrossRef]

12. Tunç, C. Asymptotic stability and boundedness criteria for nonlinear retarded Volterra integro-differential equations. J. King Saud Univ. Sci. 2016, 30, 3531-3536. [CrossRef]

13. Tunç, C. Stability and boundedness in Volterra-integro differential equations with delays. Dyn. Syst. Appl. 2017, 26, 121-130.

14. Tunç, O. On the qualitative analyses of integro-differential equations with constant time lag. Appl. Math. Inf. Sci. 2020, 14, 57-63.

15. Tunç, C.; Tunç, O. New results on the stability, integrability and boundedness in Volterra integro-differential equations. Bull. Comput. Appl. Math. 2018, 6, 41-58.

16. Tunç, C.; Tunç, O. New qualitative criteria for solutions of Volterra integro-differential equations. Arab J. Basic Appl. Sci. 2018, 25, 158-165. [CrossRef]

17. Tunç, C.; Tunç, O. A note on the qualitative analysis of Volterra integro-differential equations. J. Taibah Univ. Sci. 2019, 13, 490-496.

18. Vasundhara, D.J.; Mc Rae, F.A.; Drici, Z. Variational Lyapunov method for fractional differential equations. Comput. Math. Appl. 2012, 64, 2982-2989.

19. Wang, K. Uniform asymptotic stability in functional-differential equations with infinite delay. Ann. Differ. Equ. 1993, 9, 325-335. 
20. Wang, Q. The stability of a class of functional differential equations with infinite delays. Ann. Differ. Equ. 2000, $16,89-97$.

21. Wang, Y.; Li, T. Stability analysis of fractional-order nonlinear systems with delay. Math. Probl. Eng. 2014. [CrossRef]

22. Wen, Y.; Zhou, X.F. Zhang, Z.; Liu, S., Lyapunov method for nonlinear fractional differential systems with delay. Nonlinear Dyn. 2015, 82, 1015-1025. [CrossRef]

23. Zhou, B. Egorov, A.V. Razumikhin and Krasovskii stability theorems for time-varying time-delay systems. Autom. J. IFAC 2016, 71, 281-291. [CrossRef]

24. Agarwal, R.; Bohner, M.; Domoshnitsky, A.; Goltser, Y. Floquet theory and stability of nonlinear integro-differential equations. Acta Math. Hung. 2005, 109, 305-330. [CrossRef]

25. Hristova, S.; Tunç, C. Stability of nonlinear Volterra integro-differential equations with Caputo fractional derivative and bounded delays. Electron. J. Differ. Equ. 2019, 30, 11.

26. Hristova, S.; Tunç, C. On the stability properties of retarded Volterra integro-fractional differential equations with Caputo derivative. AIP Conf. Proc. 2021, 2321, 030013.

27. Du, X.T. Some kinds of Liapunov functional in stability theory of RFDE. Acta Math. Appl. Sin. 1995, 11, 214-224. [CrossRef]

28. Hale, J.K.; Verduyn Lunel, S.M. Introduction to functional-differential equations. In Applied Mathematical Sciences; Springer: New York, NY, USA, 1993; p. 99.

29. Razumihin, B.S. On stability of systems with retardation. Russ. Prikl. Mat. Meh. 1956, 20, 500-512.

30. Razumihin, B.S. The application of Lyapunov's method to problems in the stability of systems with delay. Automat. Remote Control 1960, 21, 515-520.

31. Agarwal, R.; Hristova, S.; O'Regan, D. Lyapunov functions and stability of Caputo fractional differential equations with delays. Differ. Equ. Dyn. Syst. 2020, in press. [CrossRef]

32. Agarwal, R.; O’Regan, D.; Hristova, S. Stability of Caputo fractional differential equations by Lyapunov functions. Appl. Math. 2015, 60, 653-676. [CrossRef]

33. Hu, J.-B.; Lu, G.-P.; Zhang, S.-B.; Zhao, L.-D. Lyapunov stability theorem about fractional system without and with delay. Commun . Nonlinear Sci. Numer. Simul. 2015, 20, 905-913. [CrossRef]

34. Li, Y.; Chen, Y.Q.; Podlubny, I. Stability of fractional-order nonlinear dynamic systems: Lyapunov direct method and generalized Mittag-Leffler stability. Comput. Math. Appl. 2010, 59, 1810-1821. [CrossRef]

35. Liu, S.; Jiang, W.; Li, X.; Zhou, X.-F. Lyapunov stability analysis of fractional nonlinear systems. Appl. Math. Lett. 2016, 51, 13-19. [CrossRef]

36. Noeiaghdam, S.; Sidorov, D. Caputo-Fabrizio fractional derivative to solve the fractional model of energy supply-demand System. Math. Model. Eng. Probl. 2020, 7, 359-367. [CrossRef]

37. Podlubny, I. Fractional differential equations. An introduction to fractional derivatives, fractional differential equations, to methods of their solution and some of their applications. In Mathematics in Science and Engineering; Academic Press, Inc.: San Diego, CA, USA, 1999; p. 198.

38. Duarte-Mermoud, M.A.; Aguila-Camacho, N.; Gallegos, J.A.; Castro-Linares, R. Using general quadratic Lyapunov functions to prove Lyapunov uniform stability for fractional order systems. Commun. Nonlinear Sci. Numer. Simul. 2015, 22, 650-659. [CrossRef] 